\title{
A review on the impact of lung cancer multidisciplinary care on patient outcomes
}

\author{
Monique Y. Heinke ${ }^{1}$, Shalini K. Vinod $^{1,2}$ \\ ${ }^{1}$ Liverpool and Macarthur Cancer Therapy Centre, Liverpool, NSW, Australia; ${ }^{2}$ South Western Sydney Clinical School, University of New South \\ Wales, NSW, Australia \\ Contributions: Conception and design: All authors; (II) Administrative support: None; (III) Provision of study materials or patients: None; \\ (IV) Collection and assembly of data: All authors; (V) Data analysis and interpretation: All authors ; (VI) Manuscript writing: All authors; (VII) Final \\ approval of manuscript: All authors. \\ Correspondence to: Professor Shalini K. Vinod. Liverpool Cancer Therapy Centre, Locked Bag 7103, Liverpool BC, NSW 1871, Australia. \\ Email: Shalini.Vinod@health.nsw.gov.au.
}

\begin{abstract}
International guidelines recommend a multidisciplinary approach to the management of lung cancer due to the complexity of both patients and their disease and the multiple treatment options available. This care can be provided through patient discussion at multidisciplinary meetings where relevant medical and allied health staff formulate a consensus management plan taking all factors into consideration. This model can be extended further to include multidisciplinary clinics where the patient is present for assessment and discussion. However, conducting regular multidisciplinary meetings or clinics has significant time, resource and financial costs and therefore, it is important to assess the impact of multidisciplinary care. We aimed to review published evidence, from 2000 to 2019, to evaluate the impact of multidisciplinary care on lung cancer outcomes. There were 29 studies found, 11 evaluating multidisciplinary clinics, 14 studying multidisciplinary meetings and four where the model of care was not defined. There was only one randomised trial and three prospective studies, the remainder being retrospective studies. Despite limitations in trial design and confounding factors, overall, multidisciplinary care in lung cancer was associated with improvements in patient outcomes, in particular improved survival for all stages of lung cancer. Lung cancer patients managed in a multidisciplinary setting were more likely to receive active treatment and had improved utilisation of all treatment modalities: surgery, radiotherapy and chemotherapy. In addition, the treatment recommendations were more likely to be consistent with lung cancer management guidelines. These improved outcomes support the recommendations for a multidisciplinary approach to lung cancer care.
\end{abstract}

Keywords: Multidisciplinary care; lung cancer; survival; treatment utilisation; guideline adherence

Submitted Jul 31, 2019. Accepted for publication Oct 28, 2019.

doi: $10.21037 /$ tlcr.2019.11.03

View this article at: http://dx.doi.org/10.21037/tlcr.2019.11.03

\section{Introduction}

Lung cancer management is challenging due to the underlying biological diversity of the disease and the complexity of its patient population. Many factors, including stage, histology, age, comorbidities, symptoms, patient performance status and preference impact on management. Active treatment options include surgery, radiation therapy and systemic therapy in the form of chemotherapy, targeted therapies or immunotherapy. Frequently a combination of two or three modalities may be used. In patients with advanced disease and those with significant comorbidities or poor performance status a palliative approach to treatment may be most appropriate. The multiplicity of treatment options requires close collaboration between multiple specialists in order to develop a management plan based on evidence and tailored to individual patients. 
This collaboration is the cornerstone of multidisciplinary care. Multidisciplinary care refers to medical care that is provided by a number of different medical and allied health specialities, who meet regularly to prospectively share, weigh and synthesise information relating to individual patients. Following discussion, a collective decision regarding the most appropriate management options, including the need for further investigations or treatment, is made. In lung cancer multidisciplinary care is provided by radiation oncologists, medical oncologists, thoracic surgeons, respiratory physicians, radiologists, pathologists, nuclear medicine physicians, and palliative care. Other health professionals may also be involved, including nursing, social workers and pharmacists.

Multidisciplinary care as part of lung cancer management has been recommended in a number of international management guidelines in the USA (1-3), the UK (4), Australia (5), and France (6). Multidisciplinary cancer care is one of the ten goals outlined in the Quality Cancer Care Statement by the American Society of Clinical Oncology (ASCO)-the European Society for Medical Oncology (ESMO) (3), which states the "Optimal treatment of cancer should be provided by a team that includes, where appropriate, multidisciplinary medical expertise composed of medical oncologists, surgical oncologists, radiation oncologists, palliative care experts, as well as oncology nurses and social workers. Patients should also bave access to counselling for their psychological, nutritional, and other needs." The Australian National Service Improvement Framework for Cancers outlines the need to develop strategies to encourage participation by clinicians and health care providers in multidisciplinary care (7). The National Comprehensive Cancer Network (NCCN) guidelines for the management of lung cancer specifically, recommend the diagnosis and treatment of lung cancer should be based on a consensus of specialists in a multidisciplinary setting $(1,2)$. In addition, patient assessment in a multidisciplinary setting is a published key performance indicator (KPI) for lung cancer in some jurisdictions $(8,9)$.

There are several models of multidisciplinary care delivery. One model is a regular multidisciplinary meeting (MDM), case conference, or tumour board where a quorum of treating specialists attend a regular meeting to present and discuss individual patients and make decisions on patient care. MDMs may be held as a face-to-face meeting or as a virtual or teleconferenced meeting if distance is prohibitive $(10,11)$. A second model of care is a multidisciplinary clinic (MDC) where a centralised lung cancer clinic is established, and patients are able to see the appropriate specialists within this clinic. An MDM is frequently arranged either prior to or following the MDC.

Measuring the impact lung multidisciplinary care has on patient outcomes is difficult, due to the lack of randomised controlled trials and multiple confounding factors. Patients reviewed in a multidisciplinary setting are younger and tend to have early stage disease, fewer comorbidities, and better performance status (12-16). Patient age, comorbidity score, cancer stage and service volume of the hospital are factors associated with multidisciplinary care (14). It can be difficult to separate out the effects of multidisciplinary care from those related to patient or institutional factors.

A number of different studies have sought to assess the impact of multidisciplinary care in terms of outcomes for lung cancer patients. A multitude of outcomes have been assessed in these studies, including the impact of multidisciplinary care on survival; treatment utilisation; adherence to guideline treatment; the timeliness of treatment; referral to palliative care; quality of life (QOL); patient satisfaction; and enrolment in clinical trials. This review summarises the trials published, from 2000 to 2019, which examine the impact of multidisciplinary care on lung cancer outcomes (Table 1).

\section{Impact on survival}

The evidence for the impact of multidisciplinary care on lung cancer survival is varied, ranging from a statistically significant improved survival for patients who were managed via an MDM or MDC (12-15,26,28,30,35,36); to a non-significant trend towards improved survival (24); and finally no survival benefit in patients $(10,17,27,37)$.

One study randomised patients with a possible diagnosis of lung cancer to workup at a centralised tertiary centre MDM or to conventional workup locally. There was no difference in survival between patients randomised to the two arms either overall or for patients receiving curative treatment (27). This study was limited by a small sample size and approximately one third of patients having a noncancer diagnosis. A retrospective review of lung cancer patients at a single institution also found discussion at an MDM had no impact on survival for patients with lung cancer [odds ratio (OR) 1.0: 95\% CI, 0.86-1.17] (10). Given that over $80 \%$ of patients had locally advanced or metastatic disease in both groups, this finding is consistent with expected survival outcomes in this cohort. Another retrospective study found no difference when comparing the median survival pre- and post-establishment of an 
Table 1 Summary of studies reporting the impact of multidisciplinary care in lung cancer on patient outcomes

\begin{tabular}{|c|c|c|c|c|c|c|c|c|c|}
\hline Reference & Patient cohort and study design & Study design & Nature of multidisciplinary care & Study limitations & Survival & Treatment utilisation & Timeliness & $\begin{array}{l}\text { Guideline } \\
\text { treatment }\end{array}$ & Other outcomes \\
\hline \multicolumn{10}{|c|}{ Studies evaluating the impact of MDC } \\
\hline $\begin{array}{l}\text { Riedel } \\
\text { et at. (2006) } \\
\text { USA (17) }\end{array}$ & $\begin{array}{l}\mathrm{N}=345 \\
\text { NSCLC patients, 1999-2003 } \\
244 \text { MDC + MDM patients, } 101 \text { MDM-alone } \\
\text { patients (NB-this group was from the time } \\
\text { following cessation of the MDC. Patients were } \\
\text { discussed at an MDM) }\end{array}$ & $\begin{array}{l}\text { Retrospective pre- and } \\
\text { post-test auditt }\end{array}$ & $\begin{array}{l}\text { MDC + weekly MDM attended by respiratory } \\
\text { physicians, medical oncologists, and radiation } \\
\text { oncologists at a single Veteran Affairs institution } \\
\text { MDM was attended by the specialists involved in } \\
\text { the MDC as well as thoracic surgeons, radiologist, } \\
\text { and pathologist } \\
\text { Following cessation of the MDC, the weekly MDM } \\
\text { continued }\end{array}$ & $\begin{array}{l}\text { Retrospective trial design } \\
\text { Male only population }\end{array}$ & $\begin{array}{l}\text { No difference in 1-year } \\
\text { survival in the MDC and } \\
\text { MDM-alone patients (i.e., } \\
\text { seen subsequent to the } \\
\text { cessation of the MDC) }\end{array}$ & NR & $\begin{array}{l}\text { Similar time to diagnosis } \\
\text { and time to treatment for } \\
\text { patients seen in the MDC } \\
\text { and MDM-alone }\end{array}$ & NR & $\begin{array}{l}\text { Clinical trials-increased } \\
\text { proportion of MDC patients } \\
\text { enrolled on clinical trials } \\
\text { compared to MDM-alone } \\
\text { patients }\end{array}$ \\
\hline $\begin{array}{l}\text { Conron } \\
\text { et al. } \\
\text { (2007) } \\
\text { Australia } \\
\text { (18) }\end{array}$ & $\begin{array}{l}\text { N=431 } \\
\text { Lung cancer patients, Sep. 2002-Sep. } 2004 \\
\text { No comparison group }\end{array}$ & $\begin{array}{l}\text { Retrospective cohort, } \\
\text { no comparison group }\end{array}$ & $\begin{array}{l}\text { Weekly MDC + MDM at a single institution. } \\
\text { Thoracic surgery, respiratory physician, medical } \\
\text { oncologist, radiation oncologist, radiologist, } \\
\text { pathologist, palliative care physician, lung cancer } \\
\text { nurse coordinator }\end{array}$ & $\begin{array}{l}\text { Retrospective study } \\
\text { Single institution } \\
\text { Referral bias } \\
\text { Registry data } \\
\text { No comparison group }\end{array}$ & NR & NR & $\begin{array}{l}\text { Treatment (surgery, } \\
\text { radiotherapy or } \\
\text { chemotherapy) started } \\
\text { within recommended } \\
\text { timeframe as outlined in } \\
\text { international guidelines }\end{array}$ & NR & NR \\
\hline $\begin{array}{l}\text { Seek } \\
\text { et al. } \\
\text { (2007) } \\
\text { USA (19) }\end{array}$ & $\begin{array}{l}\text { Patient numbers not reported } \\
\text { All lung cancer patients } \\
\text { Timeframe not reported }\end{array}$ & $\begin{array}{l}\text { Retrospective pre- and } \\
\text { post-test cohort study }\end{array}$ & $\begin{array}{l}\text { Fortnightly MDC + MDM at a single institution. } \\
\text { Thoracic surgery, respiratory physician, } \\
\text { medical oncologist, radiation oncologist, nurse } \\
\text { practitioners }\end{array}$ & $\begin{array}{l}\text { Retrospective study } \\
\text { Patient numbers and time frame of study not } \\
\text { reported } \\
\text { No comparison group to assess impact of } \\
\text { patient satisfaction }\end{array}$ & NR & NR & $\begin{array}{l}\text { Time from diagnosis } \\
\text { to treatment improved } \\
\text { following establishing the } \\
\text { MDC }\end{array}$ & NR & $\begin{array}{l}\text { Patient satisfaction-high } \\
\text { levels of patient satisfaction } \\
\text { with MDC }\end{array}$ \\
\hline $\begin{array}{l}\text { Bjegovich- } \\
\text { Weidman } \\
\text { et al. } \\
\text { (2010) } \\
\text { USA (20) }\end{array}$ & $\begin{array}{l}\mathrm{N}=46 \\
\text { Lung cancer MDC patients between 2007-2009 } \\
\text { Comparison group - patients diagnosed pre-MDC, } \\
\text { numbers not given }\end{array}$ & $\begin{array}{l}\text { Retrospective pre- and } \\
\text { post-test study }\end{array}$ & $\begin{array}{l}\text { MDC every third week attended by surgeon, } \\
\text { respiratory physicians, medical oncologist, } \\
\text { radiation oncologist, and a care coordinator at a } \\
\text { regional hospital }\end{array}$ & $\begin{array}{l}\text { Retrospective trial design } \\
\text { Small numbers } \\
\text { Comparison group not clearly defined }\end{array}$ & NR & NR & $\begin{array}{l}\text { Reduction in time to } \\
\text { curative treatment } \\
\text { following establishing the } \\
\text { MDC }\end{array}$ & NR & $\begin{array}{l}\text { Patient satitfaction-high } \\
\text { levels of patient satisfaction } \\
\text { in both the pre- and post- } \\
\text { MDC groups }\end{array}$ \\
\hline $\begin{array}{l}\text { Horvath } \\
\text { et al. (2010) } \\
\text { USA (21) }\end{array}$ & $\begin{array}{l}\text { Patient numbers not reported } \\
\text { Patients seen in lung and gynaecology MDC } \\
\text { Timefrrame not reported }\end{array}$ & $\begin{array}{l}\text { Retrospective pre- and } \\
\text { post-test cohort study }\end{array}$ & $\begin{array}{l}\text { Weekly MDC + MDM at a single institutition. } \\
\text { Cardiothoracic surgery, respiratory physician, } \\
\text { medical oncologist, radiatition oncologist, palliative } \\
\text { care physician, allied health and nurse practititioner }\end{array}$ & $\begin{array}{l}\text { Retrospective study } \\
\text { Patient numbers and time frame of study not } \\
\text { reported } \\
\text { No comparison group to assess impact of } \\
\text { patient satisfaction }\end{array}$ & NR & NR & $\begin{array}{l}\text { Improved time from } \\
\text { MDC to treatment (for } \\
\text { surgery, chemotherapy, } \\
\text { radiotherapy) compared } \\
\text { to the time taken prior to } \\
\text { establishing the MDC }\end{array}$ & NR & $\begin{array}{l}\text { Palliative care-improved } \\
\text { rate of referral to palliative } \\
\text { care following the setup } \\
\text { with the MDC }\end{array}$ \\
\hline $\begin{array}{l}\text { Smith } \\
\text { et al. (2012) } \\
\text { UK (22) }\end{array}$ & $\begin{array}{l}\text { N=497 } \\
\text { NSCLC patients seen in the palliative care clinic, } \\
\text { Jan } 2009-J a n 2011 \\
\text { No comparison group }\end{array}$ & $\begin{array}{l}\text { Qualitative study, } \\
\text { abstract only }\end{array}$ & $\begin{array}{l}\text { Weekly MDC attended by respiratory physicians, } \\
\text { thoracic oncologist, palliative care physician, lung } \\
\text { cancer nurse, and clinical trials }\end{array}$ & $\begin{array}{l}\text { Retrospective design } \\
\text { No comparison group } \\
\text { Abstract only } \\
\text { Qualitative study }\end{array}$ & NR & NR & NR & NR & $\begin{array}{l}\text { Patient satisfaction- } \\
\text { improved service provision, } \\
\text { reduced referal time, } \\
\text { reduced transport costs, } \\
\text { smooth transition between } \\
\text { services } \\
\text { Clinic trials-improved } \\
\text { access to clinical trials }\end{array}$ \\
\hline $\begin{array}{l}\text { Kedia } \\
\text { et al. } \\
\text { (2015) } \\
\text { USA (23) }\end{array}$ & $\begin{array}{l}\mathrm{N}=46 \\
\text { Focus groups for patients with lung cancer their } \\
\text { carers diagnosed seen within or outside of the } \\
\text { MDC. Mar } 2013-J a n \text { 2014 } \\
22 \text { patients, } 24 \text { carers }\end{array}$ & $\begin{array}{l}\text { Prospective post-test } \\
\text { qualitative study with } \\
\text { comparison group }\end{array}$ & $\begin{array}{l}\text { Weekly MDC attended by thoracic surgeon, } \\
\text { respiratory physician, medical oncologist, } \\
\text { radiology support, and a nurse coordinator }\end{array}$ & $\begin{array}{l}\text { Small sample size } \\
\text { Single institution } \\
\text { Comparison group not clearly defined }\end{array}$ & NR & NR & NR & NR & $\begin{array}{l}\text { Patient satisfaction- } \\
\text { patients and care } \\
\text { givers preferred the } \\
\text { multidiscipininary approach, } \\
\text { improved eficiency, } \\
\text { improved coordination and } \\
\text { communication in the MDC }\end{array}$ \\
\hline
\end{tabular}


Table 1 (continued)

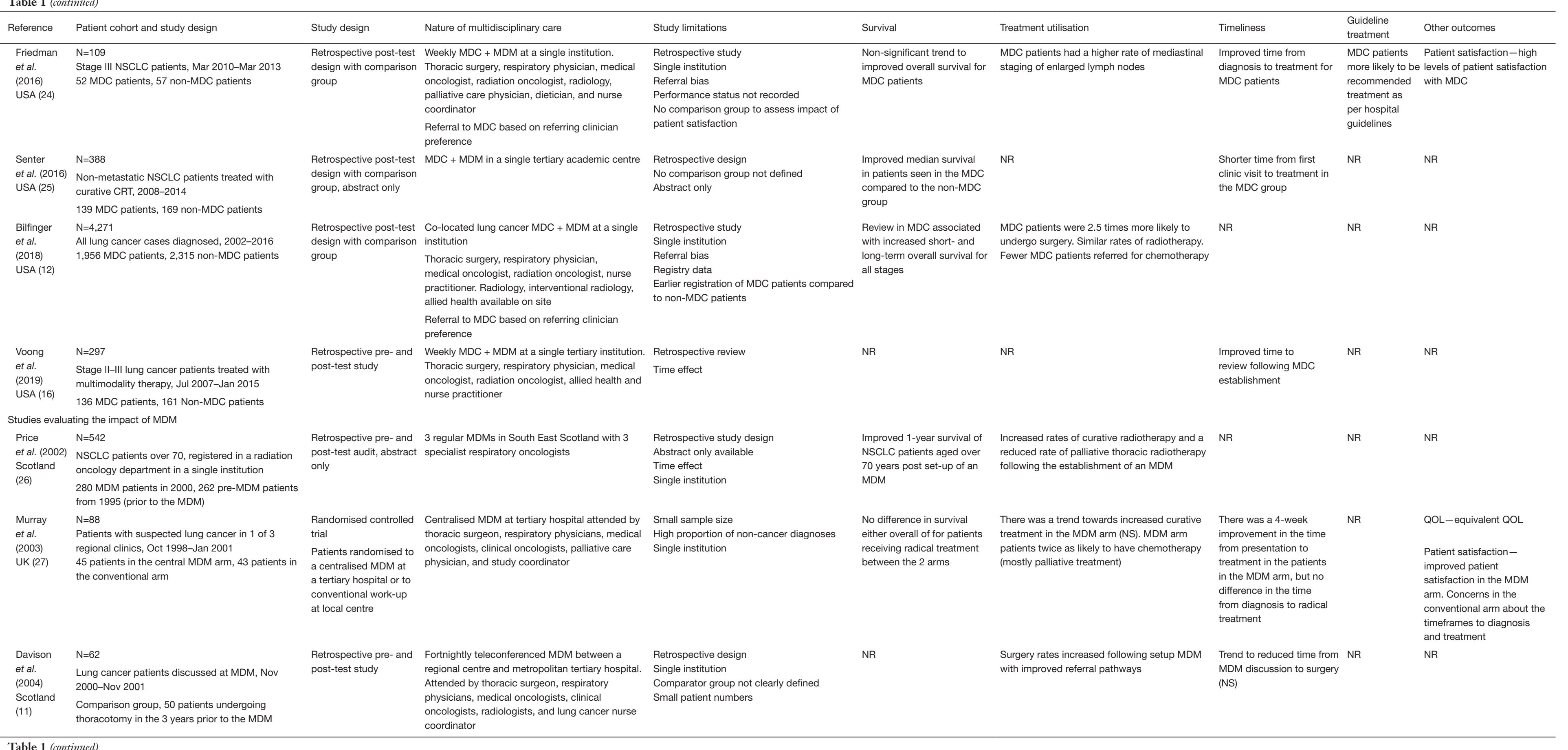


Table 1 (continued)

\begin{tabular}{|c|c|c|c|c|c|c|c|c|c|}
\hline Reference & Patient cohort and study design & Study design & Nature of multidisciplinary care & Study limitations & Survival & Treatment utilisation & Timeliness & $\begin{array}{l}\text { Guideline } \\
\text { treatment }\end{array}$ & Other outcomes \\
\hline $\begin{array}{l}\text { Forrest } \\
\text { et al. } \\
\text { (2005) } \\
\text { UK (28) }\end{array}$ & $\begin{array}{l}\mathrm{N}=243 \\
\text { Inoperable NSCLC (stage III/IV patients, diagnosed } \\
\text { in } 1997 \text { (pre-MDM and } 2001 \text { (post-MDM) } \\
156 \text { MDM patients, } 167 \text { pre-MDM patients }\end{array}$ & $\begin{array}{l}\text { Retrospective pre- and } \\
\text { post-test study }\end{array}$ & $\begin{array}{l}\text { An MDM consisting of with two respiratory } \\
\text { physicians, two surgeons, a medical oncologist, } \\
\text { a clinical oncologist, a palliative care physician, } \\
\text { a radiologist and a lung cancer nurse at a single } \\
\text { tertiary hospital }\end{array}$ & $\begin{array}{l}\text { Retrospective study } \\
\text { Single institution } \\
\text { Time effect } \\
\text { Stage drift }\end{array}$ & $\begin{array}{l}\text { Improved median survival in } \\
\text { the MDM patients }\end{array}$ & $\begin{array}{l}\text { MDM patients had increased rates of active } \\
\text { treatment and chemotherapy. Rates of } \\
\text { radiotherapp with uurative or palliative intent } \\
\text { remained unchanged }\end{array}$ & $N R$ & NR & $N R$ \\
\hline $\begin{array}{l}\text { Stevens } \\
\text { et al. (2008) } \\
\text { NZ (29) }\end{array}$ & $\begin{array}{l}\mathrm{N}=140 \\
\text { Stage I or II NSCLC patients, } 2004 \\
81 \text { MDM, } 59 \text { non-MDM }\end{array}$ & $\begin{array}{l}\text { Retrospective post- } \\
\text { test design, with } \\
\text { comparison group }\end{array}$ & $\begin{array}{l}\text { MDM attended by cardiothoracic surgeons, } \\
\text { respiratory physicians, medical oncologists, } \\
\text { radiation oncologists, and radiologists }\end{array}$ & $\begin{array}{l}\text { Retrospective design } \\
\text { Small patient numbers } \\
\text { Referral bias }\end{array}$ & $\mathrm{NR}$ & $\begin{array}{l}\text { Patients with stage I or II disease discussed } \\
\text { at MDM were more likely to undergo curative } \\
\text { treatment }\end{array}$ & $N R$ & NR & NR \\
\hline $\begin{array}{l}\text { Bydder } \\
\text { et al. } \\
\text { (2009) } \\
\text { Australia } \\
\text { (30) }\end{array}$ & $\begin{array}{l}\text { N=98 } \\
\text { Inoperable NSCLC (stage III/IV patients, } 2006 \\
81 \text { MDM patients, } 17 \text { non-MDM patients }\end{array}$ & $\begin{array}{l}\text { Retrospective post-test } \\
\text { design with comparison } \\
\text { group }\end{array}$ & $\begin{array}{l}\text { Weekly MDM at a single institution. Attended by } \\
\text { cardiothoracic surgeon, respiratory physicians, } \\
\text { medical oncologists, radiation oncologists, } \\
\text { radiologist, nuclear medicine physician, } \\
\text { pathologist, palliative care physician and lung } \\
\text { cancer nurse }\end{array}$ & $\begin{array}{l}\text { Retrospective study } \\
\text { Single institution } \\
\text { Referral bias } \\
\text { Small number of patients in non-MDM group }\end{array}$ & $\begin{array}{l}\text { Improved overall survival in } \\
\text { MDM patients }\end{array}$ & $\begin{array}{l}\text { MDM patients were more likely to have active } \\
\text { treatment, and more likely to receive palliative } \\
\text { radiotherapy than non-MDM patients, Non- } \\
\text { MDM patients were more likely to receive BSC }\end{array}$ & NR & NR & NR \\
\hline $\begin{array}{l}\text { Freeman } \\
\text { et al. } \\
\text { (2010) } \\
\text { USA (31) }\end{array}$ & $\begin{array}{l}\mathrm{N}=1,222 \\
\text { Lung cancer patients, Jan } 2001-\mathrm{Dec} 2007 \\
6877 \mathrm{MDM} \text { patients (aftet setur of MDM), } 535 \text { pre- } \\
\text { MDM (seen prior to setup of MDM) }\end{array}$ & $\begin{array}{l}\text { Retrospective pre-and } \\
\text { post-test study }\end{array}$ & $\begin{array}{l}\text { Bi-monthly MDM at a single institution attended } \\
\text { by thoracic surgeons, respiratory physicians, } \\
\text { medical oncologists, radiation oncologists, and a } \\
\text { radiologist }\end{array}$ & $\begin{array}{l}\text { Retrospective study } \\
\text { Single institution } \\
\text { Time effect }\end{array}$ & $N R$ & $\begin{array}{l}\text { Following the setup of the lung MDM, rates } \\
\text { of curative surgery rates were unchanged } \\
\text { of apart from stage IIIA where there was an } \\
\text { increased surgery rate; more patients received } \\
\text { neoadjuvant chemotherapy and there were } \\
\text { improved rates of complete preoperative } \\
\text { staging }\end{array}$ & $\begin{array}{l}\text { Improved time from review } \\
\text { to treatment following the } \\
\text { establishment of the lung } \\
\text { MDM }\end{array}$ & $\begin{array}{l}\text { MDM patients } \\
\text { were more } \\
\text { likely to receive } \\
\text { treatment as } \\
\text { per NCCN } \\
\text { Guidelines }\end{array}$ & $\begin{array}{l}\text { Palliative care-MDM } \\
\text { patients had trend to } \\
\text { increased referral to } \\
\text { pallititive care (NS) } \\
\text { Clinical Trials--trend to } \\
\text { increased enrolment in } \\
\text { clinical trials (NS) for MDM } \\
\text { patients }\end{array}$ \\
\hline $\begin{array}{l}\text { Vinod } \\
\text { et al. } \\
(2010) \\
\text { Australia } \\
\text { (32) }\end{array}$ & $\begin{array}{l}\mathrm{N}=335 \\
\text { Newly diagnosed lung cancer patients, Dec 2005- } \\
\text { Dec } 2007\end{array}$ & $\begin{array}{l}\text { Retrospective database } \\
\text { review. No comparison } \\
\text { group }\end{array}$ & $\begin{array}{l}\text { Weekly teleconferenced MDM at two institutions. } \\
\text { Attended by cardiothoracic surgeon, respiriatory } \\
\text { physicians, medical oncologists, radiatition } \\
\text { oncologists, radiologist, nuclear medicine } \\
\text { physician, pathologist, palliative care physician } \\
\text { and lung cancer nurse }\end{array}$ & $\begin{array}{l}\text { Retrospective study } \\
\text { Referral bias }\end{array}$ & $\mathrm{NR}$ & $\mathrm{NR}$ & $\begin{array}{l}\text { High rate of patients } \\
\text { discussed at MDM } \\
\text { recommended guideline } \\
\text { treatment }\end{array}$ & NR & $N R$ \\
\hline $\begin{array}{l}\text { Boxer } \\
\text { et al. } \\
\text { (2011) } \\
\text { Australia } \\
\text { (10) }\end{array}$ & $\begin{array}{l}\text { N=988 } \\
\text { NSCLC patients diagnosed, Dec 2005-Dec } 2008 \\
504 \text { MDM patients, } 484 \text { non-MDM patients }\end{array}$ & $\begin{array}{l}\text { Retrospective post-test } \\
\text { design with comparison } \\
\text { group }\end{array}$ & $\begin{array}{l}\text { Weekly teleconoferenced MDM at two institutions. } \\
\text { Attended by cardiothoracic surgeon, respiratory } \\
\text { physicians, medical oncologists, radiation } \\
\text { oncologists, radiologist, nuclear medicine } \\
\text { physician, pathologist, palliative care physician } \\
\text { and lung cancer nurse }\end{array}$ & $\begin{array}{l}\text { Retrospective study } \\
\text { Small number of institutions } \\
\text { Referral bias } \\
\text { Lack of data on performance status }\end{array}$ & $\begin{array}{l}\text { MDM discussion did not } \\
\text { impact survival }\end{array}$ & $\begin{array}{l}\text { Increased rates of chemotherapy and } \\
\text { radiotherapy for MDM patients, and equivalent } \\
\text { rates of surgery }\end{array}$ & $\begin{array}{l}\text { MDM patients had a longer } \\
\text { time to surgery (NS), } \\
\text { radiotherapy (NS), and } \\
\text { palliative chemotherapy } \\
\text { (Sig.). Equivalent time to } \\
\text { palliative radiotherapy and } \\
\text { curative chemotherapy in } \\
\text { both groups }\end{array}$ & $N R$ & $\begin{array}{l}\text { Palliative care refeerrals- } \\
\text { increased referral rate in the } \\
\text { MDM group with a longer } \\
\text { time to referral (NS) }\end{array}$ \\
\hline $\begin{array}{l}\text { Loh } \\
\text { et al. (2012) } \\
\text { NZ (33) }\end{array}$ & $\begin{array}{l}\mathrm{N}=161 \\
\text { Patients receiving radiotherapy for lung cancer, } \\
\text { Jan-Aug } 2009 \\
110 \text { MDM patients, } 51 \text { non-MDM patients }\end{array}$ & $\begin{array}{l}\text { Retrospective post-test } \\
\text { design with comparison } \\
\text { group }\end{array}$ & $\begin{array}{l}\text { Biweekly MDM held to cover different districts } \\
\text { either face-to-face or video-conferenced. Both } \\
\text { meetings were attended by thoracic surgeons, } \\
\text { respiratory physiciains, medical oncologists, } \\
\text { radiation oncologists, radiologist, at a single } \\
\text { institution }\end{array}$ & $\begin{array}{l}\text { Retrospective study } \\
\text { Referral bias }\end{array}$ & NR & NR & $\begin{array}{l}\text { MDM patients had a longer } \\
\text { time from diagnosis to } \\
\text { commencing treatment }\end{array}$ & NR & NR \\
\hline $\begin{array}{l}\text { Ung } \\
\text { et al. } \\
\text { (2016) } \\
\text { Australia } \\
\text { (34) }\end{array}$ & $\begin{array}{l}\text { N=68 } \\
\text { Lung cancer patients discussed at the MDM, } \\
\text { March-May } 2011\end{array}$ & $\begin{array}{l}\text { Prospective qualitative } \\
\text { study } \\
\text { No comparison group }\end{array}$ & $\begin{array}{l}\text { Weekly lung cancer MDM attended by thoracic } \\
\text { surgeons, respiratory physicians, medical } \\
\text { oncologists, radiation oncologists, radiologist, } \\
\text { nuclear medicine physician, nurse coordinator, } \\
\text { and allied health at a single institution }\end{array}$ & $\begin{array}{l}\text { Single institution } \\
\text { No comparator group } \\
\text { Small patient numbers } \\
\text { Short study timeframe }\end{array}$ & $N R$ & NR & $\mathrm{NR}$ & NR & $\begin{array}{l}\text { Treatment } \\
\text { recommendation- } 58 \% \\
\text { of patients had a change } \\
\text { in their management } \\
\text { plan following discussion } \\
\text { at the MDM. The MDM } \\
\text { recommended plan was } \\
\text { implemented in } 72 \% \text { cases }\end{array}$ \\
\hline
\end{tabular}


Table 1 (continued)

\begin{tabular}{|c|c|c|c|c|c|c|c|c|c|}
\hline Reference & Patient cohort and study design & Study design & Nature of multidisciplinary care & Study limitations & Survival & Treatment utilisation & Timeliness & $\begin{array}{l}\text { Guideline } \\
\text { treatment }\end{array}$ & Other outcomes \\
\hline $\begin{array}{l}\text { Rogers } \\
\text { etal. } \\
\text { (2017) } \\
\text { Australia } \\
\text { (15) }\end{array}$ & $\begin{array}{l}\mathrm{N}=593 \text { (lung cancer patients) } \\
\text { All patients diagnosed with cancer, 2009-2012 } \\
386 \text { MDM patients, } 207 \text { non-MDM patients }\end{array}$ & $\begin{array}{l}\text { Retrospective pre- and } \\
\text { post-test study }\end{array}$ & $\begin{array}{l}\text { Weekly lung cancer MDM attended by surgeon, } \\
\text { medical oncologist, radiation oncologist, } \\
\text { pathologist and radiologist. May also be attended } \\
\text { by allied health and GP }\end{array}$ & $\begin{array}{l}\text { Retrospective study } \\
\text { Single institution }\end{array}$ & $\begin{array}{l}\text { Presentation at MDM prior } \\
\text { to treatment associated } \\
\text { with reduced mortality on } \\
\text { multivariate analysis }\end{array}$ & $\begin{array}{l}\text { Patients presented at MDM were more likely } \\
\text { to undergo active treatment, including surgery } \\
\text { and concurrent CRT and less likely to have } \\
\text { single modality treatment of chemotherapy or } \\
\text { radiotherapy alone }\end{array}$ & NR & NR & NR \\
\hline $\begin{array}{l}\text { Stone } \\
\text { et al. } \\
\text { (2018) } \\
\text { Australia } \\
\text { (35) }\end{array}$ & $\begin{array}{l}\mathrm{N}=1,197 \\
\text { Lung cancer patients diagnosed or treated, Jan } \\
2006 \text {-Dec } 2012 \\
295 \text { MDM patients, } 902 \text { non-MDM patients }\end{array}$ & $\begin{array}{l}\text { Retrospective cohort } \\
\text { study }\end{array}$ & $\begin{array}{l}\text { Weekly lung cancer MDM attended by full-range } \\
\text { of medical sub-specialties } \\
\text { Referral to MDM by treating physician at a single } \\
\text { institution }\end{array}$ & $\begin{array}{l}\text { Retrospective study } \\
\text { Single institution } \\
\text { Referral bias } \\
\text { Performance status not recorded }\end{array}$ & $\begin{array}{l}\text { Improved survival in the MDM } \\
\text { group at } 1,2 \text { and } 5 \text { years } \\
\text { for all stages, except stage } \\
\text { IIIB. Improved survival in the } \\
\text { MDM group at } 5 \text { years based } \\
\text { on multivariate analysis }\end{array}$ & NR & NR & NR & $\begin{array}{l}\text { Palliative care referral- } \\
\text { no difference in palliative } \\
\text { care referrals for stage IV } \\
\text { patients, with a longer time } \\
\text { to referral in MDM group }\end{array}$ \\
\hline $\begin{array}{l}\text { Tamburini } \\
\text { et al. } \\
\text { (2018) } \\
\text { Italy (36) }\end{array}$ & $\begin{array}{l}\text { N=477 } \\
\text { NSCLC patients managed with surgery, Jan 2008- } \\
\text { Dec } 2015 \\
231 \text { MDM patients, } 246 \text { patients seen prior to } \\
\text { establishing the MDM }\end{array}$ & $\begin{array}{l}\text { Retrospective pre- and } \\
\text { post-test audit }\end{array}$ & $\begin{array}{l}\text { Weekly lung cancer MDM attended by surgeon, } \\
\text { medical oncologist, radiation oncologist, nuclear } \\
\text { medicine physician, pathologist, radiologist and } \\
\text { lung cancer coordinator }\end{array}$ & $\begin{array}{l}\text { Retrospective cohort } \\
\text { Single institution } \\
\text { Time effect }\end{array}$ & $\begin{array}{l}\text { 1-year survival was } \\
\text { significantly improved in the } \\
\text { MDM group compared to } \\
\text { the pre-MDM patients. MDM } \\
\text { discussion an independent } \\
\text { prognostic factor on } \\
\text { multivariate analysis }\end{array}$ & $\begin{array}{l}\text { MDM patients were more likely to have } \\
\text { complete preoperative staging. No difference } \\
\text { in the resection margins, postoperative } \\
\text { complications or postoperative mortality }\end{array}$ & NR & NR & NR \\
\hline \multicolumn{10}{|c|}{ Studies evaluating the impact of multidisciplinary care-model of care not specified } \\
\hline $\begin{array}{l}\text { Keating } \\
\text { et al. (2013) } \\
\text { USA (37) }\end{array}$ & $\begin{array}{l}\mathrm{N}=24,616 \text { (lung cancer) } \\
\text { Cancer patients recorded with the Department of } \\
\text { Veterans Affairs Central Cancer Registry, 2001- } \\
2004\end{array}$ & $\begin{array}{l}\text { Retrospective audit of } \\
\text { cancer registry }\end{array}$ & $\begin{array}{l}\text { One of more MDM (tumour board), either general } \\
\text { or lung cancer specific MDM }\end{array}$ & $\begin{array}{l}\text { Retrospective audit of cancer registry } \\
\text { Referral bias }\end{array}$ & $\begin{array}{l}\text { No difference in } 1 \text { year all- } \\
\text { cause survival for NSCLC } \\
\text { or SCLC regardless of } \\
\text { discussion at MDM or not }\end{array}$ & $\begin{array}{l}\text { Unresected stage I/II patients discussed at a } \\
\text { general MDM more likely to have radiotherapy. } \\
\text { Patients discussed at a general or lung cancer } \\
\text { MDM were more likely to nundergo curative } \\
\text { intent CRT for stage IIIA NSCLC or limited } \\
\text { stage SCLC. There was no difference in the } \\
\text { rates of curative surgery for stage //IIINSCLC, } \\
\text { mediastinal evaluation, doublet chemotherapy } \\
\text { for stage IV NSCLC }\end{array}$ & NR & NR & NR \\
\hline $\begin{array}{l}\text { Kehl } \\
\text { etal. }(2015) \\
\text { USA (38) }\end{array}$ & $\begin{array}{l}\mathrm{N}=2,132 \\
\text { Lung cancer patients linked to a survey of } 1,601 \\
\text { treating oncologists, } 2003-2005\end{array}$ & $\begin{array}{l}\text { Prospective patterns } \\
\text { of care observational } \\
\text { study }\end{array}$ & MDM participation & Referral bias & NR & $\begin{array}{l}\text { Stage I/II NSCLC patients more likely to } \\
\text { undergo curative surgery if surgeon participated } \\
\text { in weekly lung cancer-specific MDMs }\end{array}$ & NR & NR & $\begin{array}{l}\text { Patient satisfaction-MDM } \\
\text { discussion did not impact } \\
\text { on patient satisfaction } \\
\text { or patient impression of } \\
\text { communication } \\
\text { Clinical Trials - patients } \\
\text { discussed at centres with } \\
\text { weekkly MDMs were more } \\
\text { likely to be enrolled onto } \\
\text { clinical trials }\end{array}$ \\
\hline $\begin{array}{l}\text { Pan } \\
\text { et al. (2015) } \\
\text { Taiwan } \\
\text { (14) }\end{array}$ & $\begin{array}{l}\mathrm{N}=32,569 \\
\text { Newly diagnosed NSCLC identified from National } \\
\text { Cancer Registry, 2005-2011 } \\
\text { 4,632 MDM patients, 27,937 Non-MDM patients }\end{array}$ & $\begin{array}{l}\text { Retrospective audit } \\
\text { review }\end{array}$ & $\begin{array}{l}\text { Hospitals participating in "Multidisciplinary Cancer } \\
\text { Treatment Team" according to the Taiwanese } \\
\text { "Cancer Centers for a Great Improvement in } \\
\text { Quality of Cancer Care" initiative }\end{array}$ & $\begin{array}{l}\text { Retrospective audit of cancer registry } \\
\text { Referral bias } \\
\text { Time effect }\end{array}$ & $\begin{array}{l}\text { Improved 2-year survival } \\
\text { for all stages. MDT status } \\
\text { predictor of survival on } \\
\text { multivariate analysis. MDT } \\
\text { participants (most apparent } \\
\text { for stages III/I disease) had } \\
\text { a significant reduction in the } \\
\text { hazard ratio for death }\end{array}$ & NR & NR & NR & NR \\
\hline
\end{tabular}

MDC, multidisciplinary clinic; MDM, multidisciplinary meeting; NR, not reported; NSCLC, non-small cell lung cancer; SCLC, small cell lung cancer; CRT, chemoradiotherapy; QOL, quality of lif; NS, not significant; sig., significant; BSC, best supportive care; GP, general practitioner. 
MDC (1.2 vs. 1.0 years, $\mathrm{P}=0.39)$ (17). A patterns of care study linking the presence of MDMs to cancer registry data found there was no difference in 1-year survival for non-small cell lung cancer (NSCLC) or small cell lung cancer (SCLC) in centres with a lung cancer specific MDM, a general MDM or no MDM (37).

Other studies have reported improved survival associated with multidisciplinary care. A retrospective audit of a state-wide cancer registry found patients discussed at MDM had improved overall survival (10.8 vs. 5.5 months, $\mathrm{P}<0.001)$ (13). A retrospective analysis of all newly diagnosed cancer patients in a regional cancer service found discussion at a lung cancer MDM was associated with reduction in mortality [hazard ratio (HR) $0.72,95 \%$ CI: $0.60-0.88, \mathrm{P}<0.01$ ] after adjusting for age, tumour stage, any treatment and number of comorbidities (15). For elderly NSCLC patients, aged over 70 years, referred for radiotherapy, 1-year survival improved from $18.3 \%$ to $23.5 \%(\mathrm{P}=0.049)$ following the setup of the MDM (26).

Several studies have evaluated the impact of multidisciplinary care by stage of cancer $(12,14,24,28,30,35,36)$. Some studies have evaluated a particular stage only $(24,28,30,36)$ whilst others have studied the impact of stage within a cohort of patients of all stages $(24,28,30,36)$.

Tamburini et al. found improved 1-year survival for earlystage NSCLC patients who underwent curative resection (82\% pre-MDM vs. $92 \%$ post-MDM, $\mathrm{P}=0.006$ ) (36). This improvement may be associated with improved pre-operative staging in these patients, which was an independent prognostic factor in this study (36). Friedman et al. found a non-significant improvement in median overall survival for patients with stage III NSCLC from 14 to 17 months $(\mathrm{P}=0.054)$ for patients not reviewed and reviewed at an MDC respectively (24). This study was limited by small patient numbers, no comparison of treatment modalities or details on patient performance status or comorbidities. In inoperable stage III and IV NSCLC, MDM discussion was associated with improved survival in two retrospective single institution trials $(28,30)$. Bydder et al. found that inoperable NSCLC patients presented at the lung cancer MDM had significantly improved median survival (349 vs. 182 days, $\mathrm{P}=0.049$ ) (30). However, patients presented at the MDM were more likely to have stage III disease, compared to more patients with stage IV disease in the non-MDM group. Forrest et al. found improved median survival in inoperable stage III/IV NSCLC patients following establishment of an MDM at a single centre (6.6 vs. 3.2 months, $\mathrm{P}<0.001)$ (28).
The distribution of patients with stage III and IV disease was similar for the two groups, however the overall numbers of patients was relatively small. Neither study included information regarding patient performance status or comorbidities.

The impact of multidisciplinary care on survival for individual specified stages of lung cancer has been assessed within larger cohorts of patients of all stages $(12,14,35)$. Bilfinger et al. conducted a retrospective review of 4,271 patients comparing patients seen in a lung cancer MDC to those not referred to the MDC (12). There was significantly improved survival for each stage at 1, 3, 5 and 10 years (apart from stage III NSCLC at 10 years). A referral bias was noted with $40 \%$ of the MDC group having stage I disease, compared to only $14 \%$ of the non-MDC group. To account for this difference, a propensity matched analysis was performed. This demonstrated significantly improved survival at 1,3 and 5 years in the MDC patients. Stone et al. demonstrated improved survival for MDM patients at 1,2 and 5 years for all stages, apart from stage IIIB at 1 and 2 years (35). On multivariate analysis after adjusting for age, gender, performance status, pathology, stage and year of diagnosis, MDM discussion remained a predictor of survival at 5 years (HR $0.7,95 \% \mathrm{CI}: 0.58-0.85, \mathrm{P}<0.001)$. This study again had differences in the stage and performance status of the MDM and non-MDM groups. An analysis of the Taiwanese National Cancer Registry found 2-year survival for patients with NSCLC for all stages was better for patients managed through a multidisciplinary model (14). There were small but significant reduction in mortality rates in the multidisciplinary care patients with stage III and IV disease (HR 0.87, 95\% CI: 0.84-0.90, $\mathrm{P}<0.001$ ). For stage I and II patients the reduced hazard for death was not significant.

On multivariate analysis, discussion at MDM remained an independent prognostic factor for improved survival $(13,15,36)$. After adjusting for tumour stage, comorbidities, age and treatment received patients discussed at MDM prior to treatment had reduced mortality compared to patients not discussed at the MDM, (HR 0.62, 95\% CI: $0.50-0.76, \mathrm{P}<0.01)(15)$.

There is inconsistent evidence for the impact of multidisciplinary care on lung cancer survival. Despite many studies demonstrating a significant improvement in survival for patients managed through an MDC or MDM, these studies are limited by retrospective and varied trial design, small patient numbers and difficulties in accounting for confounding factors which impact survival in this patient 
group. However, the largest studies which have accounted for potential imbalances in prognostic factors between MDM and non-MDM groups using statistical methods, have all shown significantly improved survival in the group receiving multidisciplinary care.

\section{Impact on treatment utilisation}

The purpose of an MDM/MDC is to develop an appropriate management plan for individual patients. Several studies have evaluated the impact of an MDM on the management of lung cancer and found the MDM recommended a change in management for $39-58 \%$ of cases compared with the referring clinician's pre-recorded management plan $(34,39,40)$. The main reasons were to arrange further investigations, a change in the recommended treatment modality or a change in treatment intent $(34,41)$. Adherence to implementation of the recommended management plan ranged between $26-98 \%(34,39,40)$.

Patients discussed in a multidisciplinary setting are more likely to receive active treatment including all treatment modalities $(13,15,20,27,29,30)$. In the only randomised trial, there was no significant difference in the use of curative treatment although patient numbers were small (27). In retrospective studies, curative treatment was twice as likely to be recommended to patients discussed at an MDM $(44 \%$ vs. $22 \%, \mathrm{P}<0.001)(13)$. Stage I/II NSCLC patients were more likely to undergo curative treatment if they were discussed at an MDM (75\% vs. $24 \%, \mathrm{P}<0.001)$ (29).

In the reverse scenario, patients not discussed at the MDM were more likely to receive best supportive care only (44\% vs. 58\%, $\mathrm{P}=0.045)$ (28). This difference may reflect the fact that the non-discussed patients generally have poorer performance status, are older and have more advanced disease $(10,13,35)$.

Patients are more likely to stay within the local system for their lung cancer treatment following the establishment of a lung MDC. Bjegovich-Weidman et al. found that $94 \%$ of patients completed their treatment at local facilities compared to $86 \%$ of lung cancer patients in the year prior to the MDC (20).

\section{Impact on surgical utilisation}

The impact of multidisciplinary care on surgical utilisation varies between studies. Three retrospective observational studies comparing surgery rates pre- and post-establishment of an MDM found that overall, discussion at an MDM did not increase the number of patients undergoing surgery $(10,31,37)$. One cancer registry audit from a single institution found patients with stages I or II lung cancer (NSCLC and SCLC) seen in a lung MDC were 2.5 times more likely to undergo surgery (12).

Patients who were initially seen in a centre with a thoracic surgery department, were $51 \%$ more likely to undergo surgery (OR 1.51, 95\% CI: 1.16-1.97) (42) and there was a $30 \%$ increase in surgery rates following the establishment of a teleconferenced MDM between a regional and tertiary referral centre (11), presumably as this provided a more direct referral pathway for patients and clinicians. Freeman et al. found that despite equivalent rates of curative surgery overall regardless of whether a patient was reviewed in the MDM but there was an increase in the proportion of patients with stage IIIA NSCLC undergoing surgery (89\% vs. $49 \%, \mathrm{P}=0.0079$ ) (31). Regular attendance at the MDM was also associated with an increased rate of surgery for patients with stage I/II lung cancer (OR 2.9: 95\% CI: 1.3-6.8) (38).

There was an improvement in completeness in staging for newly diagnosed lung cancer following the establishment of an MDM (31). In two retrospective observational studies more patients underwent mediastinal staging prior to surgery if reviewed in an MDM $(24,36)$. However, in a review of the Department of Veterans Affairs Central Cancer Registry, multidisciplinary care did not impact the rates of mediastinal evaluation (37). There were fewer patients with pathological N2 disease following surgery if their cases were reviewed in an MDM, perhaps reflecting improved pre-operative staging, however surgical margin status was unchanged (31).

Overall, multidisciplinary case discussion may increase rates of surgery for stage I and II NSCLC, especially if the patient is reviewed in an MDC setting with access to thoracic surgery or if there is an established referral pathway for thoracic surgeon review.

\section{Impact on radiotherapy utilisation}

Boxer et al. found that radiotherapy utilisation was doubled if patients were discussed at an MDM (66\% vs. 33\%, $\mathrm{P}<0.001)(10)$. Two retrospective pre- and post-studies separated radiotherapy into curative and palliative treatment $(26,28)$. These studies found a differing effect of MDM discussion. Forrest et al. showed similar rates of curative or palliative radiotherapy in patients with inoperable NSCLC before and after the establishment of an MDM (28), 
although the total number of patients, especially those receiving curative radiotherapy was small. Price et al. found a significant increase in the rate of curative radiotherapy in elderly patients ( $3 \%$ to $12 \%, \mathrm{P}=0.004)$ after establishing an $\mathrm{MDM}$ and a corresponding fall in the rates of palliative thoracic radiotherapy (65\% to $55 \%$ ) (26).

An analysis of the Department of Veterans Affairs Central Cancer Registry compared radiotherapy utilisation for unresected stage I/II NSCLC, stage IIIA NSCLC and for limited stage SCLC based on presentation at a lung cancer specific MDM, a general MDM or no MDM discussion (37). Discussion at a general MDM resulted in an increase in radiotherapy for unresected stage I or II NSCLC, (70.8\% vs. $66.5 \%, \mathrm{P}=0.04)$. Similarly, patients were significantly more likely to undergo concurrent chemoradiotherapy for stage IIIA NSCLC or limited stage SCLC if they were discussed in an MDM (either lung cancer specific or general MDM) compared to those patients not discussed at an MDM.

On multivariate analysis, discussion at MDM as well as age and tumour stage were independent predictors of treatment modality (10). Despite limitations in the studies, overall, MDM discussion leads to more patients receiving radiotherapy, both curative and palliative.

\section{Impact on chemotherapy utilisation}

For lung cancer patients overall, discussion at an MDM resulted in more patients being recommended chemotherapy $(10,28,31)$ and specifically neoadjuvant chemotherapy (31). There was no difference in adjuvant chemotherapy usage for stage IIIA disease (37). The majority of patients referred for chemotherapy had inoperable stage III $(28)$ or stage IV disease $(28,31)$ and there was no difference in the proportion of patients receiving chemotherapy in stages IIIB or earlier stage disease (31). Murray et al. demonstrated a similar trend, with patients randomised to the central MDC arm twice as likely to receive chemotherapy ( $44 \%$ vs. $23 \%, \mathrm{P}=0.03$ ) the majority being with palliative intent (27), although the patient numbers in this study were small. A review of cancer registry data demonstrated a non-significant improvement in the rates of doublet chemotherapy for patients with stage IV disease who were discussed at an MDM (37).

Multidisciplinary care of lung cancer patients is likely to be associated with an increase in the recommendation for chemotherapy overall. The timeframe for all trials published to date was prior to the use of targeted therapies or immunotherapy, therefore this was not assessed at an outcome of multidisciplinary care.

\section{Impact on guideline treatment}

There are a number of evidence-based clinical practice guidelines for the management of lung cancer $(1,2,4,5)$, which help with decisions regarding treatment pathways. The impact of a multidisciplinary discussion on guideline treatment has been addressed in several studies $(24,31,32)$. These all found greater adherence to guideline management for patients discussed in a multidisciplinary setting. A significantly greater proportion of patients with stage III NSCLC who were assessed in an MDC were managed as per hospital guidelines $(89 \%$ vs. $35 \%, \mathrm{P}<0.001)$ (24). For lung cancer patients of all stages, treatment was recommended in concordance with NCCN Guidelines in $97 \%$ after a MDM was established compared to $81 \%$ before (31).

Vinod et al. found $71 \%$ of management plans discussed at the lung MDM were concordant with guideline treatment (32). This varied according to treatment modality, $88 \%$ for guideline radiotherapy, $77 \%$ for guideline chemotherapy and $58 \%$ for guideline surgery. Reasons for not offering guideline therapy included physician decision, comorbidity, and technical factors that precluded the safe delivery of radiotherapy (32).

Despite the limitations of these studies, including their retrospective single institution design and potential referral bias, overall, multidisciplinary care of lung cancer patients results in recommendations consistent with evidence-based treatment guidelines and greater adherence to guidelinebased treatment.

\section{Impact on timeliness of care}

Timeliness of care is important in the management of lung cancer as longer time to commencing treatment is associated with the potential for disease progression as demonstrated on several studies which have performed serial positron emission tomography (PET) scans (43-45). Multiple different measures of timeliness of care have been evaluated, including the time from diagnosis to treatment $(10,17,19,20,24,27,31,33)$, time from MDM or MDC to treatment $(6,21,24,25,27,30)$, time between referral and clinic review (17), and the time from first visit to diagnosis (17).

The impact of a multidisciplinary approach for the time to treatment varies across the studies. Seven studies noted an improvement in the time to treatment for patients seen 
in an MDC (16,19-21,25,27,31). Two studies found no change in time from diagnosis to treatment, either from radiological diagnosis (24) or endobronchial ultrasound (EBUS) (17), and another two studies found a nonsignificant longer time from diagnosis to treatment for patients presented at MDMs (10,33). For patients reviewed in an MDC, the average time from first clinic appointment to the start of treatment was improved for stage III NSCLC patients (19.9 vs. 27.3 days, $\mathrm{P}=0.04)$ (24).

In general, the studies showing an improved time between diagnosis and commencing treatment were those which involved an MDC, where patients are reviewed in a single clinic by the relevant specialists and a management plan is decided at time of the clinic review $(16,19$ $21,25,27,31)$. In contrast two studies evaluating MDM discussion both found a longer (albeit non-significant) time between diagnosis and treatment $(10,33)$. This may reflect the time taken between discussion at the MDM and assessment in a subsequent clinic to discuss treatment with the patient (33).

The impact of an MDC in terms of time to diagnosis and time to treatment was maintained even after the MDC was ceased, with continuation of an MDM or case conference (17). The median time to treatment was 21 days for patients seen in the MDC and 23 days for patients who were managed in the timeframe after the MDC was no longer functioning $(\mathrm{P}=0.38)$. Similarly, the median time to diagnosis was unchanged, $45 v s .47$ days.

The time to commencing specific treatment modalities was examined in three $(10,11,33)$ studies. There was a nonsignificant reduction in time from diagnosis to surgery in patients discussed at MDM $(10,11,33)$. There was an increase in the time to commencing radiotherapy $(10,33)$ or chemotherapy (10) in those discussed at MDM, only the time to starting palliative chemotherapy was significant (10). An increased time from referral to a clinic appointment with the oncologist may account for this difference (33).

For patients seen in an MDC the average time between a decision for active treatment to commencing therapy was less than timeframes recommended by guidelines (18). Ninety-one percent of patients underwent surgery earlier than 6 weeks post MDC and no patients waited longer than the guideline recommendation of 8 weeks. Boxer $e t$ al. and Freeman et al. both found that regardless of whether patients were discussed in a multidisciplinary setting or not, the time from diagnosis to surgery met this 8 -week recommendation $(10,31)$. The time from diagnosis to surgery for patients discussed and not discussed at the
MDM was 6 and 7.1 weeks respectively in the Boxer $e t a l$. study (10) and 2.4 and 3 weeks respectively in the Freeman et al. study (31).

The mean time for patients reviewed in an MDC to commencing radiotherapy was shorter than recommended in guidelines (18), with the time to definitive radiotherapy 22 days (guidelines $\leq 28$ days) and to palliative radiotherapy was 8.4 days (guidelines $\leq 14$ days). Other studies have shown slightly longer times to the commencement of radiotherapy (intent not specified), 27 days (6) and 31 days (33).

The utility of timeliness of care to measure the impact of multidisciplinary care is not straight forward. Intuitively, a shorter time between diagnosis and treatment would reduce the risk of disease progression in early stage disease (43-45). However, timeliness of care and patient survival have been shown to have an inverse relationship. Patients who are symptomatic or have advanced disease may commence palliative radiotherapy, which tends to be a simpler technique in a shorter timeframe than for curative treatments which require a longer time for planning (46). In stage III and IV NSCLC there was improved survival associated with a shorter time from diagnosis to treatment, but no association between shorter time to treatment and survival benefit in early stage NSCLC (47).

Overall, the impact of multidisciplinary care on timeliness of care is difficult to assess as there is a wide variation in time intervals measured and most studies do not distinguish between different treatment modalities, nor treatment intent. Patients seen in an MDC setting appear to have shorter times to treatment commencement.

\section{Impact on palliative care referrals}

The importance of early referral to palliative care in patients with stage IV NSCLC has been demonstrated in a randomised controlled trial where early palliative care review was associated with a 2.3 -month improvement in median survival as well as QOL (48). The impact of lung cancer multidisciplinary care on palliative care referrals is mixed. Stone et al. found no difference in the proportion of patients with stage IV lung cancer patients referred to palliative care services regardless of discussion at MDM or not (35). Boxer at al. found a greater proportion of patients who were discussed at the MDM were referred to palliative care (10). This difference was significant and discussion at the MDM was an independent predictor of palliative care referral. A third study found an increased proportion of patients in the MDM group who were 
referred to palliative or hospice care, although this difference was not significant (31).

In an MDC setting, all patients requiring palliative care review were seen by the palliative care service compared to only $88 \%$ of patients prior to establishing the clinic (21). Patients identified a number of advantages in co-locating palliative care services within an MDC, including improved service provision, reduction in referral times, reduced transport costs and a smooth transition between services (22).

It should also be noted that patients who were discussed at an MDM had a longer time to referral to palliative care $(10,35)$. The differences in the time to palliative care referrals may reflect the fact that patients discussed in the multidisciplinary setting tend to have earlier stage disease, be younger, better performance status $(10,12-16,35)$, and are more likely to receive active treatment $(13,15,30)$.

\section{Impact on QOL}

QOL as measured by the EORTC QLQ-30 was determined for patients randomised to the centralised MDC and local care at baseline and 6 weeks (27). Overall QOL was no different between the two arms, although there was a significant reduction in certain domains (role, social and financial function) in the centralised MDC arm at 6 weeks. At the 6 -week mark, 44\% of the MDC group had commenced chemotherapy, compared to $23 \%$ in the locally treated group. Treatment-related side-effects may account for the differences in QOL seen between the two groups.

High levels of distress have been recorded in lung cancer patients (49). In addition, patient-related QOL has been demonstrated to be an independent prognostic factor in patients with advanced lung cancer (50). However, few studies have examined the impact of multidisciplinary care on QOL in lung cancer or other cancers. Multidisciplinary assessment may provide an opportunity to assess patient's QOL and to screen for distress and psychosocial concerns and provide rapid referral pathways to appropriate services. The effectiveness of this referral process is related to the participating members of the multidisciplinary team.

\section{Patient satisfaction with multidisciplinary care}

Patient satisfaction with a multidisciplinary approach to lung cancer diagnosis and management was assessed in five studies: one randomised controlled trial (27), three retrospective reviews pre- and post-establishment of a multi-disciplinary clinic $(19,20,24)$ and one prospective cohort study $(23)$. In the three retrospective reviews greater than $98 \%$ of patients surveyed were satisfied to highly satisfied with the service. However, two of these studies did not assess patients seen outside the MDC $(19,24)$. Bjegovich-Weidman et al. note that $95 \%$ of patients were very satisfied with the service prior to establishing the MDC (20), suggesting the MDC may have little impact on patient satisfaction at this institution.

Patients assessed in a centralised MDC for their investigations and management were more satisfied with the service provided than patients seen in a conventional serial care approach, in particular they felt their assessment was more efficient $(23,27)$, there was better communication $(23,27)$, medical staff had greater empathy $(27)$, and their views of their illness were considered (27). Patients and their carers felt more comfortable with physicians collaborating in the decision-making process and preferred a central point of care to maximise efficiency and reduced anxiety (23). There was a non-significant trend towards improved organisation and personal experience of care in the MDC arm (27).

\section{Impact on clinical trial enrolment}

The impact of multidisciplinary care on clinical trial enrolment has not been extensively investigated and the evidence is conflicting. Riedel et al. found lung cancer patients who were seen in the MDC were more likely to be enrolled on a clinical trial (55 patients) compared to patients who were not seen in the clinic (6 patients) (17). Freeman et al. found a non-significant increase in the proportion of patients on clinical trial for the MDM group (11\% vs. 7\%, $\mathrm{P}=0.07)(31)$. Both studies are limited by small sample size and retrospective study design.

\section{Discussion}

The diagnosis and management lung cancer are complex and management may involve utilisation of multiple treatment modalities including thoracic surgery, systemic therapy, radiation therapy and palliative care. A multidisciplinary care model, either in the form of an MDC or MDM, allows for the review and discussion of individual patient cases in order to determine an appropriate management plan in a patient-centred and structured format.

The impact of multidisciplinary care in lung cancer has been extensively studied with a wide range of outcomes reported. The available evidence is limited by the lack of high-quality evidence with only a single small randomised 
controlled trial reporting the impact of a lung cancer MDC model on patient outcomes (17). The remaining evidence is from mostly retrospective reviews comparing outcomes preand post-MDC/MDM establishment or patients discussed in the multidisciplinary setting $v$ s. patients managed outside of the multidisciplinary environment. These trials use differing methods, definitions and outcome measures, making it difficult to make any direct comparisons. In addition, there are a multitude of confounders present such as heterogeneity of tumour stage, performance status, comorbidities, socioeconomic status and access to services which impact on the significance of the findings in the studies.

Despite these limitations, multidisciplinary care does appear to have a positive impact on a number of outcomes in lung cancer. In the larger cohort trials which accounted for potential confounding factors, there was a significant improvement in survival for patients managed in a multidisciplinary setting. Multidisciplinary care was also associated with higher rates of active treatment overall and increased utilisation of surgery, chemotherapy and both curative and palliative radiotherapy. This improvement in active treatment should be considered in the context of a potential referral bias, as patients who are referred for multidisciplinary care are more likely to be younger, have better performance status, fewer comorbidities and have earlier stage disease. In fact, it is important to discuss the cohort of older patients with comorbidities as multidisciplinary input is necessary to determine fitness for curative treatment particularly if multimodality treatment is being considered.

Multidisciplinary care was associated with improved adherence to guideline management, resulting in more patients being recommended to receive a treatment recommendation based on the best available evidence. Rates of concordance between the multidisciplinary recommendations and treatment delivered varies between $63-96 \%(6,33,51)$. Concordant clinical management is important as it has been shown to be significantly associated with improved median and progression free survival (51).

Despite being a reported outcome in many studies, the impact of multidisciplinary care on the timeliness of care in lung cancer is difficult to assess due to the wide variety of time intervals measured in the different studies. An MDC model of care is likely to have a shorter time from review to treatment commencement.

Access to palliative care services is improved when there is a well-defined referral pathway, for instance the colocation of palliative care physicians within the MDC allows for all patients needing input from the palliative care service to be seen in a timely fashion $(21,22)$. MDM discussion may not have an impact on the rate of palliative care referrals and the time to palliative care referral was longer for the MDM patients, reflecting the increased rate of active treatment in this group of patients.

Other outcomes such as patient QOL, patient satisfaction and enrolment on clinical trials have been assessed in only a few studies which have significant limitations. There was no clear evidence reported to suggest that multidisciplinary care improved patient's QOL or clinical trial enrolment. Overall patients appear to be satisfied with an MDC model of care, although some patients reported finding the MDC confusing (23) and did not like the repetition of their history and examination when seen by multiple practitioners within the clinic (21). These findings suggest the need to optimise the structure of the multidisciplinary care model. Having a care coordinator as a point of contact, to help navigate patients through the MDC, or to ensure appropriate follow-up is made following MDM discussion has been demonstrated to help address these issues (19).

A potential benefit of multidisciplinary care is improved health care utilisation and a reduction in the financial cost to the patient. Following the establishment of an MDC, there was a reduction in the financial cost to patients, particularly in a reduction in the cost of diagnostic investigations as well as fewer provider visits (16). Multidisciplinary care is also associated with patients having fewer visits to their general practitioner during their workup and treatment (27).

Areas for further assessment of the benefit of multidisciplinary care should be done in a prospective manner ideally across multiple institutions. Given many institutions have well established multidisciplinary processes it would be difficult to randomise patients to not receive this care. However large patient cohort studies could potentially further review the impact on treatment utilisation and survival if all prognostic factors are documented and accounted for in analysis. Other potential outcomes of multidisciplinary care in lung cancer which could be further investigated include assessment of the impact on QOL and patient reported outcomes, the benefits of screening in high risk populations and the utilisation of the newer systemic therapies including targeted therapies and immunotherapies. 


\section{Conclusions}

Despite the limitations in the quality of evidence available, multidisciplinary care in lung cancer is associated with improvements in patient outcomes, in particular improved survival, improved utilisation of all treatment modalities, and adherence to guideline management, thus supporting the recommendations of a multidisciplinary approach to lung cancer care.

\section{Acknowledgments}

Funding: None.

\section{Footnote}

Provenance and Peer Review: This article was commissioned by the Guest Editor (Emily Stone) for the series "Lung Cancer Multidisciplinary Care" published in Translational Lung Cancer Research. The article was sent for external peer review organized by the Guest Editor and the editorial office.

Conflicts of Interest: Both authors have completed the ICMJE uniform disclosure form (available at http://dx.doi. org/10.21037/tlcr.2019.11.03). The series "Lung Cancer Multidisciplinary Care" was commissioned by the editorial office without any funding or sponsorship. Dr. SKV reports personal fees from Astra Zeneca, outside the submitted work. The authors have no other conflicts of interest to declare.

Ethical Statement: The authors are accountable for all aspects of the work in ensuring that questions related to the accuracy or integrity of any part of the work are appropriately investigated and resolved.

Open Access Statement: This is an Open Access article distributed in accordance with the Creative Commons Attribution-NonCommercial-NoDerivs 4.0 International License (CC BY-NC-ND 4.0), which permits the noncommercial replication and distribution of the article with the strict proviso that no changes or edits are made and the original work is properly cited (including links to both the formal publication through the relevant DOI and the license). See: https://creativecommons.org/licenses/by-nc-nd/4.0/.

\section{References}

1. National Comprehensive Care Network. Non-small cell lung cancer. Version 5. 2019. In: NCCN: Clinical practice guidelines in oncology. 2019. Available online: https:// www.nccn.org/. Accessed 15 Jul 2019.

2. National Comprehensive Care Network. Small cell lung cancer. Version 1. 2019. In: NCCN: Clinical practice guidelines in oncology. 2019. Available online: https:// www.nccn.org/. Accessed 15 Jul 2019.

3. American Society of Clinical Oncology; European Society for Medical Oncology. ASCO-ESMO consensus statement on quality cancer care. Ann Oncol 2006;17:1063-4.

4. National Institute for Health and Care Excellence. Lung cancer: diagnosis and management. In: NICE guidance 2019. Available online: https://www.nice.org.uk/guidance. Accessed 30 Jun 2019.

5. Cancer Council Australia. Optimal care pathway for people with lung cancer. 2016. Available online: https:// www.cancer.org.au/. Accessed 30 Jun 2019.

6. Leo F, Venissac N, Poudenx M, et al. Multidisciplinary management of lung cancer: how to test its efficacy? J Thorac Oncol 2007;2:69-72.

7. National Health Priority Action Council. National service improvement framework for cancer. 2004. Available online: https://www.cosa.org.au/. Accessed 15 Jul 2019.

8. Mazzone PJ, Vachani A, Chang A, et al. Quality indicators for the evaluation of patients with lung cancer. Chest 2014;146:659-69.

9. Hermens RP, Ouwens MM, Vonk-Okhuijsen SY, et al. Development of quality indicators for diagnosis and treatment of patients with non-small cell lung cancer: a first step toward implementing a multidisciplinary, evidence-based guideline. Lung Cancer 2006;54:117-24.

10. Boxer MM, Vinod SK, Shafiq J, et al. Do multidisciplinary team meetings make a difference in the management of lung cancer? Cancer 2011;117:5112-20.

11. Davison AG, Eraut CD, Haque AS, et al. Telemedicine for multidisciplinary lung cancer meetings. J Telemed Telecare 2004;10:140-3.

12. Bilfinger TV, Albano D, Perwaiz M, et al. Survival outcomes among lung cancer patients treated using a multidisciplinary team approach. Clin Lung Cancer 2018;19:346-51.

13. Mitchell PL, Thursfield VJ, Ball DL, et al. Lung cancer in Victoria: are we making progress? Med J Aust 
2013;199:674-9.

14. Pan CC, Kung PT, Wang YH, et al. Effects of multidisciplinary team care on the survival of patients with different stages of non-small cell lung cancer: a national cohort study. PLoS One 2015;10:e0126547.

15. Rogers MJ, Matheson L, Garrard B, et al. Comparison of outcomes for cancer patients discussed and not discussed at a multidisciplinary meeting. Public Health 2017;149:74-80.

16. Voong KR, Liang OS, Dugan P, et al. Thoracic oncology multidisciplinary clinic reduces unnecessary health care expenditure used in the workup of patients with non-small-cell lung cancer. Clin Lung Cancer 2019;20:e430-41.

17. Riedel RF, Wang X, McCormack M, et al. Impact of a multidisciplinary thoracic oncology clinic on the timeliness of care. J Thorac Oncol 2006;1:692-6.

18. Conron M, Phuah S, Steinfort D, et al. Analysis of multidisciplinary lung cancer practice. Intern Med J 2007;37:18-25.

19. Seek A, Hogle WP. Modeling a better way: navigating the healthcare system for patients with lung cancer. Clin J Oncol Nurs 2007;11:81-5.

20. Bjegovich-Weidman M, Haid M, Kumar S, et al. Establishing a community-based lung cancer multidisciplinary clinic as part of a large integrated health care system: aurora health care. J Oncol Pract 2010;6:e27-30.

21. Horvath LE, Yordan E, Malhotra D, et al. Multidisciplinary care in the oncology setting: historical perspective and data from lung and gynecology multidisciplinary clinics. J Oncol Pract 2010;6:e21-6.

22. Smith J, Williamson I, Ionescu A, et al. 97 Improving outcomes for palliative lung cancer patients: experience of a multiprofessional parallel clinic model in a district general hospital [abstract]. Lung Cancer 2012;75:S32.

23. Kedia SK, Ward KD, Digney SA, et al. 'One-stop shop': lung cancer patients' and caregivers' perceptions of multidisciplinary care in a community healthcare setting. Transl Lung Cancer Res 2015;4:456-64.

24. Friedman EL, Kruklitis RJ, Patson BJ, et al. Effectiveness of a thoracic multidisciplinary clinic in the treatment of stage III non-small-cell lung cancer. J Multidiscip Healthc 2016;9:267-74.

25. Senter J, Hooker C, M L, Voong K, et al. Thoracic multidisciplinary clinic improved survival in patients with lung cancer. [Abstract]. Int J Radiat Oncol Biol Phys
2016;96:S134.

26. Price A, Kerr G, Gregor A. 237 Oral The impact of multidisciplinary teams and site specialisation on the use of radiotherapy in elderly people with non-small cell lung cancer (NSCLC). Radiother Oncol 2002;64:S80.

27. Murray PV, O'Brien ME, Sayer R, et al. The pathway study: results of a pilot feasibility study in patients suspected of having lung carcinoma investigated in a conventional chest clinic setting compared to a centralised two-stop pathway. Lung Cancer 2003;42:283-90.

28. Forrest LM, McMillan DC, McArdle CS, et al. An evaluation of the impact of a multidisciplinary team, in a single centre, on treatment and survival in patients with inoperable non-small-cell lung cancer. Br J Cancer 2005;93:977-8.

29. Stevens W, Stevens G, Kolbe J, et al. Management of stages I and II non-small-cell lung cancer in a New Zealand study: divergence from international practice and recommendations. Intern Med J 2008;38:758-68.

30. Bydder S, Nowak A, Marion K, et al. The impact of case discussion at a multidisciplinary team meeting on the treatment and survival of patients with inoperable nonsmall cell lung cancer. Intern Med J 2009;39:838-41.

31. Freeman RK, Van Woerkom JM, Vyverberg A, et al. The effect of a multidisciplinary thoracic malignancy conference on the treatment of patients with lung cancer. Eur J Cardiothorac Surg 2010;38:1-5.

32. Vinod SK, Sidhom MA, Delaney GP. Do multidisciplinary meetings follow guideline-based care? J Oncol Pract 2010;6:276-81.

33. Loh J, Stevens G, Stevens W, et al. Concordance between thoracic multidisciplinary meeting recommendations for radiation therapy and actual treatment for lung cancer. J Med Imaging Radiat Oncol 2012;56:696-702.

34. Ung KA, Campbell BA, Duplan D, et al. Impact of the lung oncology multidisciplinary team meetings on the management of patients with cancer. Asia Pac J Clin Oncol 2016;12:e298-304.

35. Stone E, Rankin N, Kerr S, et al. Does presentation at multidisciplinary team meetings improve lung cancer survival? Findings from a consecutive cohort study. Lung Cancer 2018;124:199-204.

36. Tamburini N, Maniscalco P, Mazzara S, et al. Multidisciplinary management improves survival at 1 year after surgical treatment for non-small-cell lung cancer: a propensity score-matched study. Eur J Cardiothorac Surg 2018;53:1199-204.

37. Keating NL, Landrum MB, Lamont EB, et al. Tumor 
boards and the quality of cancer care. J Natl Cancer Inst 2013;105:113-21.

38. Kehl KL, Landrum MB, Kahn KL, et al. Tumor board participation among physicians caring for patients with lung or colorectal cancer. J Oncol Pract 2015;11:e267-78.

39. Kee F, Owen T, Leathem R. Decision making in a multidisciplinary cancer team: does team discussion result in better quality decisions? Med Decis Making 2004;24:602-13

40. Schmidt HM, Roberts JM, Bodnar AM, et al. Thoracic multidisciplinary tumor board routinely impacts therapeutic plans in patients with lung and esophageal cancer: a prospective cohort study. Ann Thorac Surg 2015;99:1719-24.

41. Lamb BW, Sevdalis N, Taylor C, et al. Multidisciplinary team working across different tumour types: analysis of a national survey. Ann Oncol 2012;23:1293-300.

42. Rich AL, Tata LJ, Free CM, et al. Inequalities in outcomes for non-small cell lung cancer: the influence of clinical characteristics and features of the local lung cancer service. Thorax 2011;66:1078-84.

43. Lin P, Koh ES, Lin M, et al. Diagnostic and staging impact of radiotherapy planning FDG-PET-CT in non-small-cell lung cancer. Radiother Oncol 2011;101:284-90.

44. Everitt S, Herschtal A, Callahan J, et al. High rates of tumor growth and disease progression detected on serial

Cite this article as: Heinke MY, Vinod SK. A review on the impact of lung cancer multidisciplinary care on patient outcomes. Transl Lung Cancer Res 2020;9(4):1639-1653. doi: 10.21037/tlcr.2019.11.03 pretreatment fluorodeoxyglucose-positron emission tomography/computed tomography scans in radical radiotherapy candidates with nonsmall cell lung cancer. Cancer 2010;116:5030-7.

45. Mohammed N, Kestin LL, Grills IS, et al. Rapid disease progression with delay in treatment of non-small-cell lung cancer. Int J Radiat Oncol Biol Phys 2011;79:466-72.

46. Osarogiagbon RU. Making the evidentiary case for universal multidisciplinary thoracic oncologic care. Clin Lung Cancer 2018;19:294-300.

47. Vinod SK, Chandra A, Berthelsen A, et al. Does timeliness of care in Non-Small Cell Lung Cancer impact on survival? Lung Cancer 2017;112:16-24.

48. Temel JS, Greer JA, Muzikansky A, et al. Early palliative care for patients with metastatic non-small-cell lung cancer. N Engl J Med 2010;363:733-42.

49. Graves KD, Arnold SM, Love CL, et al. Distress screening in a multidisciplinary lung cancer clinic: prevalence and predictors of clinically significant distress. Lung Cancer 2007;55:215-24.

50. Ganz PA, Lee JJ, Siau J. Quality of life assessment. An independent prognostic variable for survival in lung cancer. Cancer 1991;67:3131-5.

51. Osarogiagbon RU, Phelps G, McFarlane J, et al. Causes and consequences of deviation from multidisciplinary care in thoracic oncology. J Thorac Oncol 2011;6:510-6. 